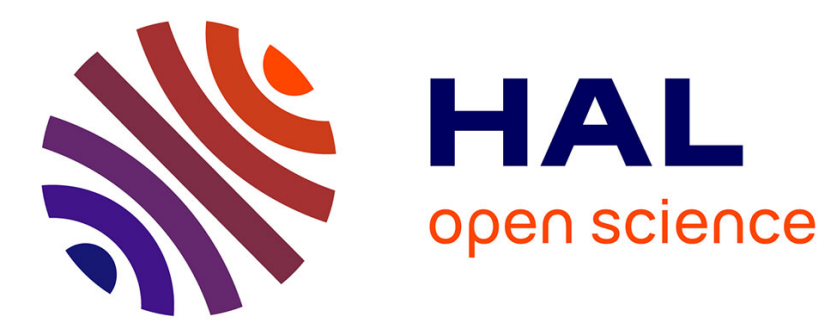

\title{
Politique agricole en France et prix de la terre \\ François Facchini
}

\section{To cite this version:}

François Facchini. Politique agricole en France et prix de la terre. Politiques et Management public, 1997, 15 (4), pp.27 - 46. 10.3406/pomap.1997.2163 . hal-00490336

\section{HAL Id: hal-00490336 https://hal.science/hal-00490336}

Submitted on 8 Jun 2010

HAL is a multi-disciplinary open access archive for the deposit and dissemination of scientific research documents, whether they are published or not. The documents may come from teaching and research institutions in France or abroad, or from public or private research centers.
L'archive ouverte pluridisciplinaire HAL, est destinée au dépôt et à la diffusion de documents scientifiques de niveau recherche, publiés ou non, émanant des établissements d'enseignement et de recherche français ou étrangers, des laboratoires publics ou privés. 
«Politique agricole et prix de la terre », Politiques et Management Public, 1997, décembrel, vol.15, numéro 4.

\title{
POLITIQUE AGRICOLE EN FRANCE ET PRIX DE LA TERRE
}

\author{
François Facchini
}

RESUME : Cet article comprend la politique agricole commune et sa réforme à partir de l'objectif de contrôle du capital foncier de la profession agricole. Les effets de la politique agricole sur le prix de la terre sont mobilisés pour corroborer cette explication.

\section{INTRODUCTION}

La croissance des coûts budgétaires de la politique agricole commune induite par la surproduction agricole, d'une part, les avancées du libre échange et l'importance des pollutions d'origine agricole, d'autre part, ont progressivement obligé les autorités publiques à engager la réforme de la politique agricole commune. Celle-ci a consisté, tout d'abord, à limiter la surproduction agricole par une politique de gel des terres et de contingentement. Elle a, ensuite, proposé un certain nombre de mesures agri-environmentales afin d'inciter les agriculteurs à produire moins de biens agricoles et plus d'aménités rurales (faune, flore, paysage, eau pure, air pur). La réforme de 1992 (entrée en application au 1 juillet 1993 pour la campagne de commercialisation) se caractérise par la mise en place d'aides directes à l'agriculteur, partiellement découplées des volumes produits, par une baisse des prix garantis (céréales et viandes ${ }^{1}$ ) et par une contraction de l'offre (gel des terres ${ }^{2}$ ). D'une politique dominée par les interventions sur le marché on est ainsi passé par une politique où les aides directes prennent de plus en plus de place. La politique agricole aujourd'hui distribue des aides compensatrices pour limiter l'impact de la baisse des prix de soutien et des aides au gel des terres pour répondre à la baisse des superficies cultivées.

Ces mesures peuvent se comprendre comme des moyens de soutenir le revenu des agriculteurs et de réaliser ainsi l'objectif de la politique agricole commune depuis sa création. Notre hypothèse complète cette explication puisqu'elle souhaite rendre compte de l'évolution de la politique agricole à partir de l'objectif de contrôle du capital foncier des agriculteurs. La terre permet à la fois de produire des biens et de se constituer un patrimoine. L'évolution de la politique agricole s'expliquerait alors par les plus-values foncières et les gains en capital captés par les agriculteurs. Une trop forte baisse de la valeur ajoutée brute des agriculteurs ferait chuter le prix de la terre et le montant du patrimoine professionnel des agriculteurs. Nous faisons ainsi l'hypothèse que l'orientation actuelle de la politique agricole s'explique aussi par des préoccupations foncières. Nous supposons, de plus, que la politique agricole s'explique par les intérêts fonciers de la profession agricole nous acceptons donc implicitement que la production de la politique agricole obéit à une logique de monopole bilatéral ministère de l'agriculture-FNSEA (Winyard [27], Balisacan et Roussamet [2]).

\section{Politique agricole de garantie des prix et prix de la terre}

Notre première tâche est de montrer que la politique agricole commune soutient les revenus des agriculteurs. Trois arguments soutiennent cette hypothèse. L'objectif politique de la politique agricole, tout d'abord, accrédite cette hypothèse. L'article 39 du Traité de Rome et l'article 1 de la loi d'orientation agricole de 1960 donnent pour mission à la politique agricole de garantir une parité de revenu avec les autres catégories sociales. La logique, ensuite, fonde cette affirmation. Si les aides directes et indirectes (garantis des prix) sont positives, les revenus agricoles hors transferts sont

\footnotetext{
${ }^{1}$ «La réduction du prix d'achat à l'intervention des céréales a été de $25 \%$ le 1 juillet 1993 puis de 6,7\% le 1 juillet 1994 et le 1 juillet 1995. Celle concernant les gros bovins a été de 5\% à ces trois mêmes dates. Par ailleurs le prix des oléagineux et des protéagineux continue à se fixer sur le marché mondial conformément au système en vigueur depuis juillet 1992 et pour les protéagineux depuis juillet 1993 (INSEE, Comptes de l'agriculture 1995, Résultats $n^{\circ} 475$, Economie générale $n^{\circ} 131$, juin 1996).

2 «Le taux de gel obligatoire a été fixé à $15 \%$ des superficies cultivées en céréales et oléo-protéagineux à partir de la campagne 1993-1994. Il a été modifié et réduit à 13,3\% pour la campagne 1995-1996 » (INSEE [1996]).
} 
«Politique agricole et prix de la terre », Politiques et Management Public, 1997, décembre; vol.15, numéro 4.

inévitablement supérieurs aux revenus sans transferts. L'existence de 145 milliards de francs en 1991 d'aides publiques à l'agriculture, enfin, comprenant 62,9 milliards de francs d'aides à l'agriculture productive, 63,7 milliards de francs d'aides au financement de la protection sociale et 15 milliards de francs d'aides aux secteurs non agricoles (ministères, recherche, etc.) là confirme (Delord et al [7]) ${ }^{3}$. Les subventions d'exploitation reçues par la branche agriculture (Graphique 1) provoquent naturellement une augmentation des revenus agricoles par rapport à une situation de marché sans interventions publiques.

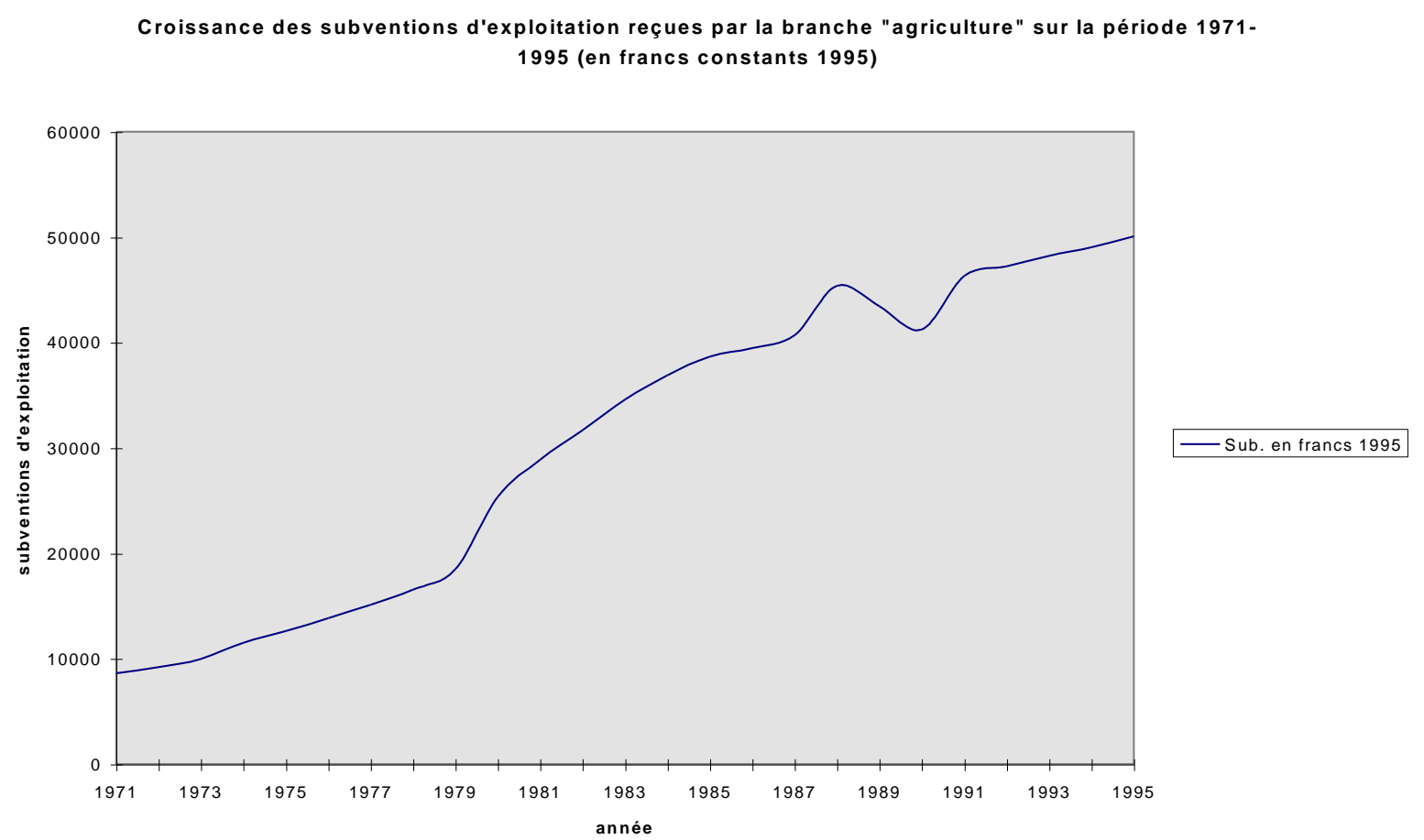

Sources: INSEE Résultats. Les comptes de l'agriculture de 1959 à 1993, Economie générale, №329$330, n^{\circ} 94-95$, octobre 1994 (valeurs en millions de francs).

Cette politique a-t-elle des effets sur le prix de la terre?

\subsection{Les déterminants économiques du prix de la terre}

Il existe «sur le prix de la terre un large consensus théorique: le prix de la terre est la capitalisation d'un revenu, fermage ou valeur de rendement procuré par un hectare de terre » (Boinon et Cavailhes [3], p.215). «La valeur vénale de la terre s'établit à la valeur actuelle de ses revenus nets futurs, toute augmentation de $1 \%$ de ceux-ci devrait selon la théorie économique, susciter une augmentation relative de même montant de la valeur vénale » (Sneessens [24], p.357). Si $\mathrm{r}$ est le taux d'actualisation et $\mathrm{R}$ la productivité marginale de la terre la valeur actuelle de la terre (VA) vaut:

(2) $\mathrm{VA}=\frac{\mathrm{R} 1}{(1+\mathrm{r})}+\frac{\mathrm{R} 2}{(1+\mathrm{r})^{2}}+\ldots+\frac{\mathrm{Rn}}{(1+\mathrm{r})}$

Dans l'hypothèse où la terre donne une durée infinie de revenus annuels constants, sa valeur s'exprime sous une forme simple. On retrouve, ainsi, la formule de capitalisation de David Ricardo $(\mathrm{P}=\mathrm{R} / \mathrm{r})$ où $\mathrm{P}$ est le prix de la terre, $\mathrm{R}$ la rente foncière et, $\mathrm{r}$ le taux d'intérêt utilisé pour la capitalisation.

\footnotetext{
${ }^{3}$ Notons que plus de $77 \%$ des financements à l'agriculture productive sont affectés à la régularisation des marchés et à l'orientation des productions (Delord et al. [7], p.190).
} 

vol.15, numéro 4.

Selon les acteurs la terre n'est cependant pas demandée pour le même usage. Pour préciser cette demande on peut distinguer trois types de valeur: la valeur de consommation, la valeur de placement et la valeur productive.

La valeur productive, tout d'abord, est fonction de la productivité marginale de la terre. La terre est un intrant nécessaire à la production industrielle, agricole ou immobilière. Toute variation du prix de la terre modifie la combinaison productive, le choix de la technique de production étant fonction de l'abondance relative des facteurs. La technologie est, dans cette perspective, une variable endogène. La fonction d'objectif ici est la maximisation du profit.

La valeur de placement, ensuite, est fonction du revenu constaté ou possible de la terre (Caziot [6], p.12) ${ }^{4}$. L'investisseur cherche à valoriser son épargne par l'achat de terre. Il en tire des loyers (fermage) et une possibilité de plus-value lors de la vente s'il anticipe une hausse du prix de la terre. La valeur de placement met ainsi en avant le motif de spéculation (Shalit et Schmitz [23], p.710). La terre devient un moyen parmi d'autre de diversifier son portefeuille et d'accroître son revenu et son capital futur. La fonction d'objectif n'est plus la maximisation du profit mais la maximisation de l'utilité dans le temps. Outre les prix des denrées agricoles qui déterminent le montant des fermages la qualité de la terre jouera aussi un rôle important dans la détermination du revenu foncier futur. L'investisseur (le bailleur) peut, pour cette raison, souhaiter améliorer le fonds afin d'augmenter les revenus futurs attendus.

La valeur de consommation, enfin, dépend de la densité de la population $\mathrm{D}$, de la proximité au centre urbain $\mathrm{P}$, de l'aspect esthétique $\mathrm{H}$ et récréatif $\mathrm{A}$, et d'autres facteurs tels que le goût ou les disponibilités de crédit X (Pope [22], p.82). La valeur de consommation s'écrit alors:

(2) $\mathrm{VC}=\mathrm{VC}(\mathrm{D}, \mathrm{P}, \mathrm{H}, \mathrm{A}, \mathrm{X})$

Empiriquement la littérature insiste plus particulièrement sur la valeur productive et la valeur locative qui n'est qu'une des composantes de la valeur de placement, délaissant quelque peu les valeurs de consommation et le motif de spéculation. J.P. Boinon et J. Cavailhès, par exemple, ([3], pp. 219-221) expliquent l'évolution du prix de la terre sur longue période par l'évolution du prix des denrées agricoles (montant des loyers) sur la période 1850-1940 et par l'évolution de la valeur ajoutée brute (VAB) par hectare (revenu) et des taux des prêts fonciers du crédit agricole pour la période 1940-1980. "Ils considèrent en effet qu'il est vain de tenter d'expliquer la baisse du prix des terres par une moindre demande pour des usages non agricoles» (Boinon et Cavaihlès [3], p.221). Le passage du prix des denrées agricoles à la VAB se justifie à leurs yeux par le fait que durant la période 1850-1940 les agriculteurs avaient un rôle directeur sur le marché foncier alors que sur la période 1940-1980 se sont les agriculteurs qui acquièrent les trois quarts des terres mises en vente. La baisse du prix de la terre (Graphique 2) s'expliquerait alors par la baisse de la VAB, par la baisse du prix des denrées agricoles et par la politique de restriction du crédit menée à partir de 1971(Boinon et Cavaihlès [3], p.226). Ces principes théoriques et ces quelques données empiriques connues, quels sont les effets de la politique agricole commune sur le prix de la terre?

\subsection{Revenu agricole anticipé, garantie des prix et prix de la terre}

La politique agricole commune (PAC) a trois types d'effets qui peuvent avoir des conséquences sur le prix de la terre: un effet revenu, un effet sur la stabilité des prix des denrées agricoles et un effet sur la prévisibilité des prix futurs. Elle a pour ces raisons modifié la valeur productive de la terre. Si l'usage de la terre est agricole, le revenu anticipé $\mathrm{R}^{*}$ dépend du prix des biens agricoles. Si la seule incertitude pour le producteur est le prix des biens agricoles (Harris [12], p.490) le niveau du revenu agricole anticipé par hectare de terre s'écrit (3) $R^{*} t=p^{*}$. Q $t-C_{t}$, avec $\mathrm{p}^{*}$ le prix anticipé des biens agricoles, Qt, la quantité de biens agricoles produite et $\mathrm{Ct}_{\mathrm{t}}$ les coûts de

\footnotetext{
${ }^{4}$ La valeur de placement est grevée par les frais de mutation, d'entretien et les charges fiscales (Alston [1]).
} 
production. La politique de garantie des prix agricoles pratiquée par les autorités européennes accroît, par conséquent, le revenu net anticipé par hectare (par rapport à ce qu'il serait sans aides indirectes) en stabilisant les anticipations sur les prix des biens agricoles qui chaque année sont réexaminés au regard des prix de marché. Toute hausse du prix des biens agricoles favorise, donc, une hausse des revenus agricoles et du prix de la terre ${ }^{5}$.

Toute hausse du prix de la terre, au regard des modèles d'innovation induite, modifie ensuite la combinaison productive puisqu'elle accroît la rareté relative de la terre. On constate, d'ailleurs, une forte intensification du système de production. Par l'augmentation des consommations intermédiaires les agriculteurs palliaient l'insuffisance de terre et préparaient la rupture de l'harmonie entre l'agriculture et l'environnement. L'intensification n'a pourtant pas limité la demande foncière des agriculteurs qui en l'absence de contraintes de débouchés avaient toujours intérêt à accroître leur production. L'agriculteur ne tenait pas compte des débouchés réels de sa production et se contentait d'accroître sa production au moindre coût afin de produire le plus possible et d'augmenter ses marges. La hausse du prix de la terre a donc provoqué une intensification des techniques de production sans baisse de la demande de terre. La PAC participe donc bien (avec les trentes années de croissance forte de la $\mathrm{VAB}$ ) à l'augmentation du prix de la terre et à cette «faim de terre » qui a dominé la France jusqu'en 1975.

En soutenant le revenu des agriculteurs la politique agricole commune suscite enfin, de la part des investisseurs, des anticipations sur la hausse régulière des prix de la terre qui leur permettent d'envisager la formation de plus-values foncières importantes. Les premiers à avoir bénéficié des plus-values foncières ont été les propriétaires fonciers non-agriculteur ensuite, sous l'influence des politiques publiques ${ }^{6}$ d'aide à l'achat de terre, ce sont les agriculteurs qui en ont bénéficié. Ainsi conformément au modèle de H. Shalit et d'A. Schmitz ([23], p.711) l'achat de terre et la demande de crédit des agriculteurs s'expliquent par leur désir d'augmenter leur revenu présent et de valoriser leur épargne (revenu futur). En complément aux études qui portent leur attention uniquement sur la valeur productive de la terre, il s'agit de mettre l'accent sur le motif de spéculation et l'importance de la terre dans la logique patrimoniale des agriculteurs.

\subsection{Capacité d'endettement des agriculteurs, plus-values foncières, prix agricoles garantis et prix de la terre}

Le raisonnement est le suivant. L'achat de terre augmente la capacité de production des exploitations, car l'intensification reste contrainte par la superficie exploitée; d'une part parce que certains investissements technologiques supposent une superficie minimale pour être viable, et d'autre part parce que pour une technologie donnée le seul moyen d'accroître sa production est d'augmenter sa surface. L'augmentation des perspectives de revenu passe ainsi par l'achat de terre. Inversement, la solvabilité de la demande foncière des agriculteurs passe par une augmentation régulière de leur revenu puisque les banques affecteront leur crédit sur la base d'une accumulation de richesse passée. En achetant de la terre l'agriculteur accroît donc à la fois ses perspectives de revenus et sa capacité d'endettement. Il augmente sa capacité de remboursement (revenu présent), dégage des perspectives d'expansion (revenu de production futur) et valorise son épargne par sa propre activité (revenu locatif et plus-values foncières à venir). La terre est pour ces raisons la garante de la modernisation, même si la logique d'investissement reste pour partie autonome, la banque accordant aussi des crédits sur des projets d'investissement strictement technique. L'investissement foncier a cependant une dimension patrimoniale qui lui donne une place centrale dans le calcul de l'agriculteur. L'achat de terre est pour l'agriculteur un moyen de capitaliser ses gains de productivité et au moment de sa cessation d'activité

\footnotetext{
${ }^{5}$ «A long terme, $10 \%$ de baisse de la valeur ajoutée brute feraient baisser le prix des terres du sixième de leur valeur» (Cavaihlès et Degoud [5], p.65).

${ }^{6}$ La politique de sélectivité du crédit menée par l'Etat à travers les prêts bonifiés distribués par le crédit agricole, les règles de reprise en faveur du preneur lors de la vente par la bailleur de sa terre, l'activité des safer, etc. illustrent bien cette politique publique en faveur des agriculteurs.
} 
soit de capter les plus-values foncières préparées par l'amélioration du fonds, soit d'augmenter son revenu par des revenus locatifs. Une fois propriétaire foncier les agriculteurs deviennent des producteurs (maximise leur profit) et des spéculateurs (maximise leur utilité dans le temps), le montant des loyers (fermage) et des plus-values foncières potentiels les intéressant autant que le montant des revenus agricoles.

En soutenant le revenu des agriculteurs et en stabilisant les anticipations des intervenants sur les marchés les politiques d'aides publiques accroissent la capacité d'épargne et d'emprunt des agriculteurs par rapport à une situation de marché sans intervention et solvabilisent leur demande foncière. Les agriculteurs ont alors raisonnablement remplacé les propriétaires bailleurs comme groupe directeur sur le marché foncier (Boinon et Cavailhès [3], p.215) et ce sont appropriés une plus grande partie des bénéfices fonciers de la politique agricole, ce qui a entretenu leur «faim de terre » au-delà de la période d'augmentation de la valeur ajoutée brute.

L'investissement foncier 1) accroît, par conséquent, le revenu anticipé des agriculteurs (accroît la superficie sans contrainte de débouchés), 2) développe leur capacité d'endettement (la terre est une garantie), 3) rentabilise leur investissement technique (économie d'échelle), 4) leur permet d'accéder plus amplement aux plus-values foncières lors de la revente et 5) valorise leur épargne (fermage). On pressent ainsi déjà que la baisse du prix de la terre ne sera pas pour la profession agricole que la traduction d'une baisse des prix des denrées agricoles ou de la VAB, elle sera aussi à l'origine d'une dévalorisation de son épargne, d'une transformation de sa combinaison productive, de moins-values foncières et d'une plus faible capacité d'endettement.

\subsection{Conclusion : excès d'offre de terre, surproduction et lâcheté de la contrainte de demande}

La PAC est cependant progressivement entré en crise parce que l'intensification des techniques de production sans contrainte de débouchés suffisamment forte a provoqué une surproduction agricole qui avait des coûts budgétaires insoutenables et incompatibles avec les exigences des accords internationaux de libre échange. En resserrant progressivement la contrainte de débouché à partir de 1984 les autorités européennes ont suscité une baisse de la demande foncière des agriculteurs. L'intensification et la contrainte de débouchés lâche entretenaient la «faim de terre ». L'intensification et le resserrement de la contrainte de débouchés au contraire développent la satiété foncière "A force de progrès technique, (...) la terre des pays riches et tempérés produit tellement qu'une forte partie en est devenue inutile» (Lamaison [14], p.19) ${ }^{7}$. A « une période de "faim de terres », au cours des années 60 et 70, succède (ainsi logiquement) une phase de "satiété foncière » dans les années 80» (Cavailhés et Degoud [5], p.62) ${ }^{8}$. La baisse des prix garantis, de la VAB, des plus-values foncières et des revenus agricoles «anticipables» expliquent alors simplement la baisse du prix de la terre qui est passé entre 1979 et 1995 (en francs constants 1995) de 39362 francs l'hectare (prix moyen pour les prairies et les terres labourables) à 18100 francs.

Ce mouvement de baisse des prix du capital foncier (Graphique 2) modifie, tout d'abord, profondément les comportements des agriculteurs qui comme nous le disions précédemment sont concernés à la fois comme investisseur et comme producteur. Il menace, ensuite, le contrôle de la terre obtenu par la profession agricole au cours des années 50-70. Une forte baisse des prix de la terre pourrait favoriser le retour des non-agriculteurs sur le marché foncier. Ces derniers profiteraient du faible prix de celle-ci pour son usage de consommation mais aussi pour développer des activités à forte intensité spatiale (Olson [22]) mais à faible rentabilité économique comme la production d'aménités rurales et les activités récréatives (tourisme rural, chasse pêche, élevage, safari, protection de la faune et de la flore, promenade, randonnées, etc.). Il suscite enfin de fortes inquiétudes sur l'équilibre des finances publiques d'une partie des communes rurales qui assure la production de

\footnotetext{
${ }^{7}$ On prévoit, d'ailleurs, dans la communauté européenne entre 1985 et l'an 2000, un surplus foncier de six millions d'hectares (Marsh et al. [19]).

${ }^{8}$ J. Cavailhès et S. Degoud ([5], p.62) remarquent cependant que la vérification de cette affirmation reste fragile.
} 

vol.15, numéro 4.

biens publics locaux principalement sur les recettes de la taxe foncière non bâtie (assise sur la valeur locative), de la mutualité sociale agricole et des chambres d'agriculture. Les effets économiques, patrimoniaux et financiers d'une baisse continue du prix de la terre pourraient expliquer pourquoi la nouvelle politique agricole commune conditionne ces aides à la superficie exploitée et augmente fortement leur montant depuis trois ans (Graphique 1) ${ }^{9}$. Reste à affiner l'analyse et à montrer que l'un des objectifs de la nouvelle PAC est de protéger les agriculteurs d'une perte de contrôle du capital foncier. A cette fin, nous présenterons le contenu de la réforme de la PAC et étudierons ces effets sur le prix de la terre.

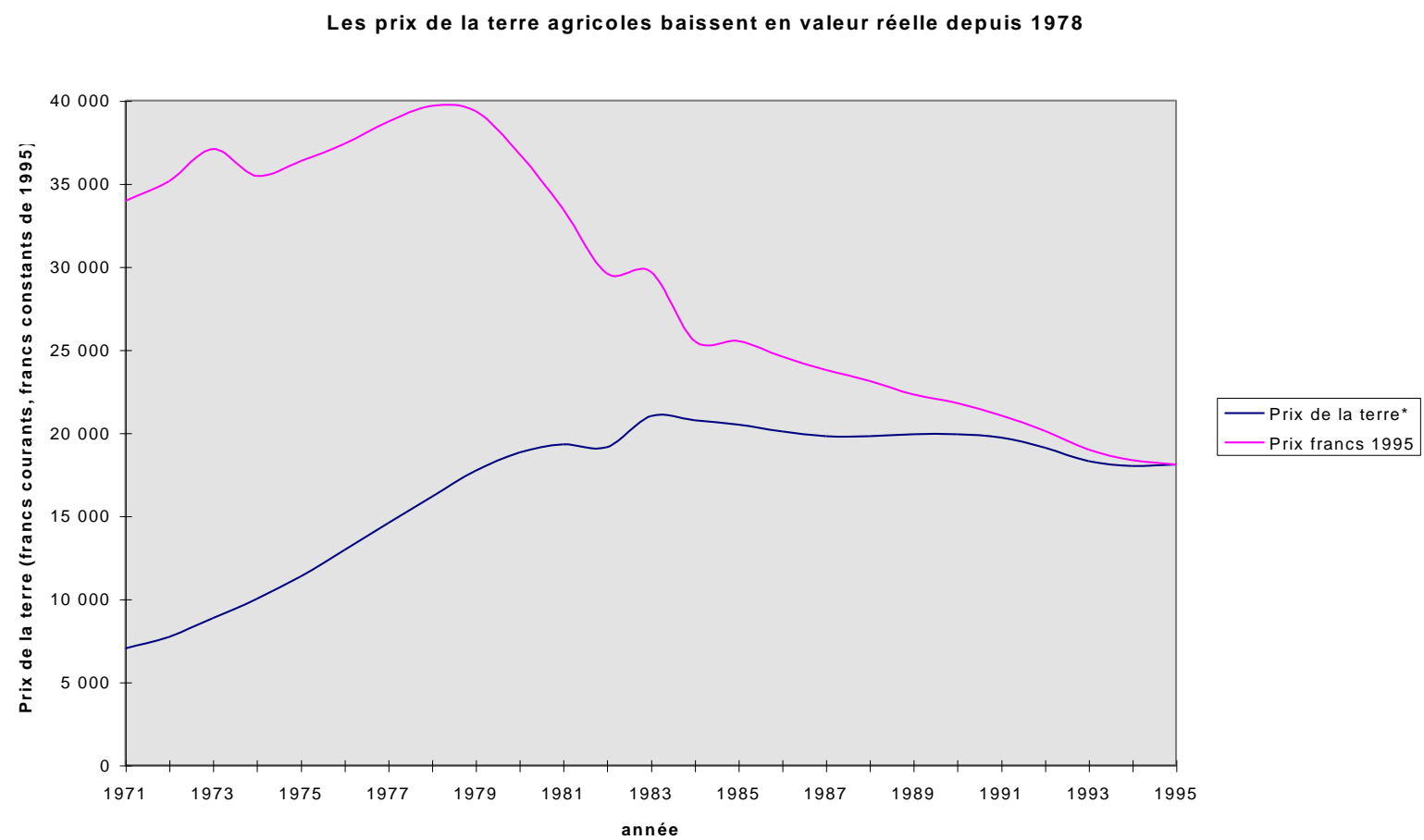

Sources: SCEES, Agreste, La statistique agricole, Données chiffrées. N84, août 1996, Le prix des terres agricoles en 1995 et Synthèses statistiques- comptes et revenus, Le prix des terres agricoles depuis 1950 (Terres labourables et prairies naturelles) Ministère de l'agriculture, avril 1984.

\section{Nouvelle politique agricole commune et prix de la terre}

La réforme de la politique agricole commune de 1992 fait écho aux premières réformes de 1984 qui avait introduit la politique des quotas sur le lait, décidé de l'extension des contrats de gestion britanniques à l'Europe par l'article 19 et mis en place les règlements concernant la politique de jachère. La politique de 1992 décide cependant de changer l'instrument de soutien au revenu des agriculteurs. Il s'agit, en effet, de développer les aides directs et de limiter le montant des prix garantis. «Les principales mesures décidées en mai 1992 sont d'une part une limitation autoritaire des quantités soutenues et d'autre part, une série de mesures incitatives à des réductions de production, notamment par la baisse des prix » (Desriers [8], p.7). Il s'agit donc d'étudier, les effets sur le prix de la terre 1) de la jachère obligatoire, 2) de la baisse du prix d'intervention sur le marché de la viande bovine et de la revalorisation des aides en la matière (aides à la vache allaitante et au bovin mâle) 3) de la baisse du prix d'intervention et des aides compensatrices sur le marché des céréales, et 4) des quotas de production.

\footnotetext{
${ }^{9}$ La progression des subventions atteint prés de 32 milliards (+19,3 milliards en 1993, +6,9 milliards en 1994 et $+5,6$ milliards en 1995).
} 
«Politique agricole et prix de la terre », Politiques et Management Public, 1997, décembre?, vol.15, numéro 4.

\subsection{Quotas laitiers, gel des terres, aide à la vache allaitante et prix de la terre}

\section{a) Prix de la terre et jachère obligatoire}

Les aides à la jachère apparaissent avec le règlement 797/85 concernant l'augmentation de l'efficacité de la politique des structures agricoles. La surface retirée de la culture doit représenter au moins $20 \%$ des terres arables de l'exploitation, et ce pour au moins cinq ans. Par ailleurs, les cultures qui sont sur les terres gelées doivent correspondre à des produits relevant d'une organisation commune de marché (OCM). Le montant de l'aide par hectare de terre retiré de la production est compris entre 700 écus et 100 écus et le budget de la communauté européenne verse entre 15 et $50 \%$ de la prime selon l'importance de celle-ci. En 1992, les autorités publiques agricoles rendent le gel des terres obligatoires. Pour compenser les pertes de revenu occasionnées par une telle mesure mais aussi par la disparition des prix de soutien pour les oléagineux et les protéagineux et la baisse des prix d'intervention pour les céréales, elles proposent des indemnités fixées depuis 1995 à l'hectare et non en tonne de céréale comme à l'origine de la mesure pour les céréales, les protéagineux et les oléagineux (Desriers [8], p.8).

Une telle politique de rationnement des terres et de baisse des prix devrait mécaniquement favoriser une baisse des prix de la terre puisqu'elle diminue le revenu net anticipé des agriculteurs. Une telle conclusion ignore cependant les comportements stratégiques des agriculteurs. La politique de gel des terres conditionne le montant des aides à la quantité de terre mise en jachère et non à la quantité produite. Les agriculteurs sont, pour cette raison, incités à acheter ou à accroître par le louage leur superficie en terres labourables afin de maintenir leur production et d'augmenter le montant des aides. Le gel des terres augmente donc le prix des terres labourables (Leathers [15], p.83). Il favorise, de plus, une forme d'intensification relativement insidieuse au regard des objectifs de qualité de l'environnement rural. "La prime à la jachère peut en effet conduire les gens avisés à planter du colza sur des terres pauvres pour les mettre ensuite en jachère et toucher la prime » (Le Cacheux et Mendras [16], p.121). Ainsi, alors qu'autrefois la politique agricole commune incitait à la production et à la modernisation, aujourd'hui elle favorise la demande de terre labourable des agriculteurs.

\section{b) Aides à la vache allaitante, prime spéciale aux bovins mâles et prix de la terre}

La nouvelle politique agricole commune a aussi engagé une politique de baisse des prix d'intervention sur le marché de la viande bovine (baisse de 5\% par an de 1993 à 1995). De la même façon que sur le marché des céréales, les autorités publiques ont cherché à compenser cette perte. Elles ont, pour cette raison, revalorisé les aides à la vache allaitantes (1396 francs par tête en 1995) et aux bovins mâles (Desriers [8], p.11). Ces primes sont modulées et plafonnées en fonction du chargement, c'est-à-dire du nombre d'animaux «primables» par hectare de surface fourragère. De la même manière, les agriculteurs peuvent souhaiter agrandir leur surface pour diminuer leur chargement et ainsi augmenter le montant des primes qui leur seront versées. La prime à l'herbe ou prime au maintien des élevages extensifs s'ajoute au niveau national à ces mesures communautaires ( 300 francs par hectare en 1995). La politique agricole favorise donc la demande de prairie et soutient le prix de ces dernières.

\section{c) Quotas laitiers et prix de la terre}

Les premiers quotas ont été instaurés sur la production laitière le 2 avril 1984 (règlements 857 et $857 \mathrm{du}$ Conseil) afin de limiter les dépenses budgétaires. Le quota de façon strictement juridique est lié à l'exploitation et non au foncier, puisqu'il n'appartient pas au bailleur en cas de cessation d'activité de son preneur. Le quota apparaît avec le producteur et disparaît avec lui. Le quota n'est transférable qu'à un autre exploitant agricole; si ce dernier demande une aide à la cessation laitière il prive l'exploitation de sa référence; si ce dernier arrête momentanément son activité il peut le garder en réserve (pas plus de deux ans); si le repreneur n'est pas un exploitant agricole elle est mise en 

vol.15, numéro 4.

réserve et gérée par le préfet (Lemonnier [17]). Le quota est donc automatiquement transféré lors de la vente des droits sur la terre (Vantreese et al. [26], p.319).

La valeur d'une exploitation laitière moderne résulte pour ces raisons plus du montant des quotas affectés à l'exploitation que de ses potentialités économiques et agronomiques ou de sa surface (Lemonnier [17], p.402). Les exploitants agricoles ont alors tendance à demander des références par le biais du foncier (Levesque, Kerisit et Oger [18], p.32), car même s'il n'est pas lié à la terre le droit de produire s'exprime en hectare. L'exploitant possède un droit à produire de 6000 ou 4500 litres par hectare selon le montant de la production laitière en 1983 (année de référence). Le droit à produire valorise donc les terres sur lequel il est affecté et dévalorise les terres qui en sont dépourvues. « $S i$ deux producteurs, l'un laitier, l'autre orienté dans une autre spéculation, sont intéressés par une parcelle disposant de quotas laitiers, chacun va déterminer un niveau de prix en fonction des anticipations de revenus de l'orientation productive de son exploitation. Dans ces conditions, une plus-value se dégagera de la transaction foncière si la valeur vénale qu'est prêt à payer le producteur de lait est supérieure à celle que peut accepter de payer le producteur engagé dans la spéculation alternative (...) Ainsi, dans une région, si la production laitière est la plus rémunératrice, la recherche de droits à produire va se matérialiser par une plus-value foncière» (Levesque et al. [18], p.33). La demande de terre des producteurs de lait dépend donc clairement des quantités de lait affecté à la parcelle ${ }^{10}$.

Plus généralement, et au regard des effets des mesures prises dans le cadre de la politique agricole depuis 1992, la demande de terre est liée à la mise en concurrence des aides publiques par l'exploitant agriculteur qui quel que soit le type de production doit agrandir son exploitation afin d'accroître ses droits à produire et ses droits à l'obtention d'une prime. Il existe bien, pour ces raisons, une valorisation publique du capital foncier qui n'est pas transférable mais est lié au producteur. La politique agricole suscite ainsi une augmentation de la demande de terre, cette dernière disparaissant derrière le producteur qui devient l'unique agent de valorisation du capital foncier. La politique agricole continue donc d'exclure les propriétaires fonciers non agricoles qui ne peuvent pas accéder aux droits à produire. Ces derniers peuvent cependant acheter les terres sans droits à produire. La superficie de ce type de terre restera malgré tout très faible, d'une part parce que perdre un quota lait ne signifie pas perdre tout droit à produire sur la terre, d'autre part parce que les intervenants sur le marché ont tendance, pour ne pas voir la valeur de placement de leur investissement s'effondrer, à répartir les droits à produire sur l'ensemble de leur capital, et enfin parce que l'agriculteur sans droit à produire peut aussi obtenir des aides agri-environnementales qui sont assises sur la superficie exploitée. C'est parce que l'agriculteur est le seul à pouvoir valoriser son capital sur fonds public qu'il exclut les autres intervenants du marché foncier, même si l'existence de terres sans quotas accessibles aux non-agriculteurs n'est pas à exclure. La nouvelle PAC répond donc toujours à une double exigence, soutenir le revenu agricole et protéger la profession agricole du retour d'investisseur foncier non agricole.

Les premiers résultats de cette politique s'expriment d'ailleurs clairement dans l'arrêt progressif de la baisse du prix des terres labourables et des prairies naturelles en 1995 (Graphique 2). En passant de 18000 francs l'hectare en 1994 à 18100 francs l'hectare en 1995 et après correction de l'inflation la baisse du prix moyen à l'hectare n'est que de $1,3 \%$, c'est la plus faible dépréciation depuis quinze ans. «Recherchée pour des besoins de surface, pour son potentiel naturel, la terre l'est aujourd'hui pour ce qu'elle supporte: quotas, droits divers, épandage, etc. » (Lemonier [17], p.400).

\footnotetext{
${ }^{10}$ En considérant qu'une parcelle dotée d'un quota de 6000 litres/ hectare se négocie à 30000 francs l'hectare se décomposant en 20000 francs pour le foncier sans quota et 1, 67 francs par litre pour le reste, le quota laitier revient à 5 francs par litre; c'est la valeur que des producteurs laitiers dépensent pour augmenter leur potentiel de production (Levesque et al. [18], p.33).
} 

vol.15, numéro 4.

\subsection{Les mesures agri-environmentales et le marché foncier}

Comme la nouvelle politique agricole commune décidée en mai 1992, les mesures agrienvironnementales ${ }^{11}$ permettent à la profession agricole de maintenir son contrôle sur le capital foncier en soutenant la demande de terre des agriculteurs qui ne peuvent augmenter le montant des transferts publics en leur faveur qu'en agrandissant la superficie de leurs exploitations (1). La spécificité de ces mesures est de maintenir l'insolvabilité de la demande environnementale des nonagriculteurs et de ralentir, ainsi, les mécanismes de redéploiement des usages du sol autour des nouveaux usages de la terre (tourisme, récréation, sport, chasse, pêche, nature, etc) (Facchini [9], p.48) (2).

\section{a) Mesures agri-environnementales et prix de la terre}

Les mesures agri-environnementales prennent leur origine dans l'article 19 du règlement CEE 797/85 modifié. Elles ont trois objectifs, protéger la qualité et à la diversité de l'espace et des produits agricoles, d'une part, afin de maintenir le potentiel et la variété agricole de l'espace rural français, organiser une politique contractuelle de gestion de l'espace rural afin de limiter la déprise agricole, d'autre part et préserver la qualité des paysages français, enfin. L'ensemble des mesures prend la forme d'aides versées sous la forme de primes à l'hectare ou à l'Unité de bétail. Elles s'adressent aux exploitants agricoles qui s'engagent à respecter un cahier des charges défini et approuvé par la Commission européenne ou les régions. Elles prennent huit formes différentes: 1) protection des eaux ${ }^{12}$, et 2) réduction d'intrants polluants, 3) reconversion des terres arables, 4) conversion en agriculture biologique ${ }^{13}, 5$ ) diminution du chargement de cheptel bovin ou ovin ${ }^{14}, 6$ ) protection des races menacées, 7) retrait de toute culture afin de protéger la faune et la flore $\left.{ }^{15}, 8\right)$ mise en place d'opération locale ${ }^{16}$. La description de chacune de ces mesures serait un travail fastidieux. A l'exception des mesures en faveur de la protection des races menacées et de la diminution du chargement de cheptel bovin ou ovin qui prévoient la distribution de primes par unité de gros bétail, les autres types de mesures sont assises sur la superficie exploitée par l'agriculteur. Les agriculteurs seront, pour cette raison, d'autant plus aidés que la taille de leur exploitation est importante.

Ce résultat permet alors de prévoir une augmentation de la demande de terre dans les années à venir, les agriculteurs étant désormais obligés de s'agrandir pour accroître le montant des aides qui leur seront affectées. Contrairement à la politique des prix garantis qui avait provoqué une course au progrès technique et un fort mouvement d'intensification sans baisse de la demande foncière du fait

\footnotetext{
${ }^{11}$ La première mesure agri-environmentale apparaît au niveau européen avec l'article 19 . Ce règlement permet d'aider les agriculteurs qui adoptent des pratiques favorables à l'environnement dans les zones sensibles du point de vue de l'environnement. Ce type a été étendu à l'ensemble du territoire par le règlement 2078/92. Sur la base de ce règlement les Etats membres de la communauté peuvent donner des aides directes à la production d'aménités rurales.

${ }^{12}$ La politique de protection des eaux consiste à lutter contre les pollutions diffuses d'origine agricole et contre l'érosion. Elle regroupe trois types d'opération, les opérations de captages (1000 francs par hectare), les opérations de protection rapprochée des cours d'eau, opération de lutte contre l'érosion ( 2500 francs par hectare).

${ }^{13}$ La reconversion en agriculture biologique est une prime qui vient en aide aux agriculteurs qui souhaitent passer d'un système de production conventionnel à un système de production biologique, les aides peuvent aller de 700 à 4700 francs par hectare pendant 2 à 3 ans selon la nature de la production.

${ }^{14}$ La diminution de la charge d'ovin ou de bovin à l'hectare permet de lutter contre les phénomènes de déprise agricole dans les zones sensibles. La collectivité verse à ce titre 1500 francs par unité de gros bovins (UGB).

${ }^{15}$ Le retrait de toute culture sert à conserver la diversité de la flore et à valoriser la faune sauvage. Il regroupe deux types d'opération, la jachère écologique qui préserve les espèces animales et végétales et la jachère faunistique qui favorise le développement des populations sauvages. Chaque opération permet d'obtenir environ 3000 francs par hectare pendant 20 ans.

${ }^{16}$ Les opérations locales cherchent à encourager les pratiques agricoles respectueuses de l'environnement. Elles distribuent, aprés acceptation du dossier, environ 100 à 1100 francs par hectare.
} 
de l'absence de contraintes de débouchés, le développement d'une politique d'aides directes, assises sur la superficie des exploitations suscitera de nouveau une faim de terre qui devrait favoriser l'extensification du moins dans l'esprit du législateur, car l'agriculteur peut toujours faire le même calcul qu'en présence des politiques de jachère, acheter ou louer des terres pour toucher les primes et intensifier sa production sur le reste de l'exploitation afin d'accroître ses bénéfices strictement agricoles. L'agriculteur respecterait d'un coté le cahier des charges sur les surfaces sous contrats qui dans une situation sans aides n'auraient pas trouvé d'usage agricole du fait du progrès technique et intensifierait de l'autre sa production sur les superficies sans contrats. La nouvelle PAC administre l'abondance sous couvert de préoccupations environnementales sans remettre en cause le productivisme de l'ancienne politique agricole. On peut d'ailleurs rappeler que lors de leur apparition en Grande-Bretagne les mesures agri-environnementales ont fait l'objet de deux nombreuses critiques. Le premier type de critique remarquait que ces mesures reposaient sur une philosophie productiviste contrariée puisque la collectivité payait le manque à gagner des agriculteurs lorsqu'ils étaient obligés de se soumettre aux lois de la protection de la nature. Le deuxième type de critique portait sur l'absence d'efficacité des mesures contractuelles, car l'agriculteur ne soumet pas toute son exploitation au contrat et les agriculteurs d'une zone ne sont pas tenus si l'un accepte le contrat de le faire, ce qui fait qu'une exploitation respectant la nature et subventionnée pour cela peut être entourée de dix exploitations «polluantes ».

\section{b) Mesures agri-environnemantales, prix de la terre et diversification des usages du sol}

Cette politique participe, aussi, à l'exclusion des non-agriculteurs du marché de la terre et renforce le monopole de la profession agricole sur l'espace rural qui au regard de son histoire a pourtant progressivement perdu son caractère exclusivement agricole. La politique agricole forme, ainsi, des barrières à l'entrée sur le marché foncier qui empêche le redéploiement des usages du sol parce qu'elle n'incite pas les individus à valoriser les valeurs de consommation de la terre. Alors que la demande foncière n'est pas seulement une demande agricole mais aussi une demande pour la consommation (VC).

En protégeant le contrôle du capital foncier par les agriculteurs, la politique agricole empêche la demande pour la consommation de s'exprimer et la diversification des usages du sol de se déployer. Elle rive les anticipations de revenu foncier à l'activité agricole alors que celle-ci grâce au progrès technique peut produire plus avec moins de terre. Elle ralentit, pour cette raison, la découverte des nouveaux usages du sol qui envahiront demain la campagne française. Elle rend artificiellement l'agriculteur irremplaçable dans sa fonction d'aménageur. Elle permet aussi aux agriculteurs de prendre une option sur les nouvelles sources de revenu de la terre (agri-tourisme, randonnées, etc.) et notamment sur la vente des aménités rurales (le paysage, la faune, la flore, la qualité de l'eau ou de l'air). Vendre la nature peut, en effet, devenir une activité économique susceptible de dégager des profits.

Une telle politique en limitant la baisse du prix de la terre et en entretenant une demande foncière artificielle désincite, cependant, les propriétaires de capitaux financiers à investir dans des activités à forte intensité spatiale. On peut, en effet, penser que l'accélération de la baisse des prix de la terre favoriserait (dans le rural profond) des investissements fonciers destinés à la récréation et à la valorisation marchande des aménités rurales. Le scénario pourrait être le suivant.

L'accélération de la baisse du prix de la terre, outre l'extensification des techniques agricoles, susciterait l'entrée sur le marché « de promoteurs environnementaux». Ainsi, au lieu de s'en remettre uniquement à la valorisation publique des aménités rurales, les autorités politiques pourraient chercher à susciter la valorisation marchande des aménités rurales, à aider les promoteurs environnementaux dans leur volonté d'investir dans le capital foncier pour développer des activités à forte intensité spatiale autour de la valorisation des aménités rurales. Il ne s'agirait plus d'inciter les agriculteurs par des mesures fiscales de type taxe- subvention à tenir compte des effets indirects de 
leur activité principale sur l'environnement, mais d'organiser le marché des aménités rurales autour des prix d'accès au territoire et/ou des prix des services permettant aux consommateurs de profiter pleinement des aménités rurales.

Si la construction d'un marché passe par la possibilité d'exclure, c'est-à-dire de faire payer les services rendus, la valorisation économique des aménités rurales passerait alors par l'organisation des droits d'accès. Organiser les droits d'accès, c'est limiter toutes les formes d'accès libre et favoriser la définition de règles capables de rendre possible la valorisation des aménités (Thomson et Whitby [25], p.305). Les propriétaires fonciers pour valoriser les aménités rurales pourraient décider de privatiser les voies de communication. Ils se donneraient, ainsi, les moyens de contrôler l'entrée et la sortie sur leur territoire et de valoriser leur patrimoine naturel par un prix d'entrée. Dans ce cas, le consommateur paierait pour consommer et le producteur adapterait et rentabiliserait son offre en fonction de la demande d'aménités rurales exprimées sur le marché. A la seule valorisation publique de la terre s'ajouterait une valorisation marchande des aménités rurales par la vente de droit d'entrée sur le territoire. Le «promoteur environnemental» organiserait, donc, sa propriété comme un parc. Il vendrait ses points de vue sur la diversité de sa faune, de sa flore ou de ses paysages. Les réserves de chasse ou de pêche, les zones de cueillettes, les espaces naturels d'excursion, la constitution de musées de la nature, les safaris photos, etc. seraient autant de moyens de valoriser les aménités rurales. Ce type de valorisation marchande des aménités rurales n'est cependant possible que lorsque le prix de la terre est bas et que la propriété foncière est faiblement morcelée ${ }^{17}$, car l'exclusion et l'organisation de l'accès sont d'autant moins coûteuses que le prix de la terre est faible et que la propriété est concentrée. Il est alors logique de penser que c'est la baisse du prix de la terre qui favoriserait l'apparition des "promoteurs environnementaux», simplement parce qu'elle baisse les coûts d'exclusion. Le faible prix de la terre ouvrirait donc le marché foncier aux activités à faible rentabilité.

Pour valoriser les aménités rurales, les «promoteurs environnementaux » pourraient aussi maintenir la gratuité de l'accès à leur territoire. Ils développeraient des activités de service telles que: l'hébergement, la protection du patrimoine architectural, la restauration, l'édition de livres (histoire régionale, flore, faune, paysage, etc) ou de cartes postales, la création d'écomusées, le développement d'emplois autour de la connaissance du milieu naturel (guides d'accueil), l'artisanat local ou les produits fermiers, etc. Le «promoteur environnemental» chercherait, ainsi, à transformer le patrimoine naturel en un support à la production de services touristiques variés (Greffe [11]). Il s'agirait de rentabiliser ces activités de service en développant l'attractivité de l'espace. Les " promoteurs environnementaux » se devraient, pour cette raison, d'entretenir la diversité écologique et culturelle de leur territoire. Contrôler le territoire par l'achat d'un droit de propriété ne serait, donc, pas suffisant il faudrait aussi trouver les moyens de le gérer à moindre coût. C'est dans ce contexte qu'il faut penser la fonction d'aménageur des agriculteurs. Ce ne serait cependant plus un contrat social mais un contrat privé ${ }^{18}$ entre un propriétaire soucieux de la qualité de l'environnement et un agriculteur. Cette différence a deux conséquences. Premièrement, la charge financière de la valorisation ne repose plus sur le budget de la nation. Deuxièmement, l'agriculture est une activité seconde par rapport à la production d'aménités rurales, elle est sous le contrôle des "promoteurs environnementaux », des associations à but non lucratifs ou plus généralement des propriétaires fonciers qui sont les donneurs d'ordre ${ }^{19}$.

\footnotetext{
${ }^{17}$ Un territoire est morcelé quand sa propriété est partagée entre beaucoup de personne.

${ }^{18}$ Le code civil considère que «le contrat est une convention par laquelle une ou plusieurs personnes s'obligent envers une ou plusieurs autres à donner à faire ou à ne pas faire quelque chose » (Code civil article 1101).

${ }^{19}$ Ce type de contrat porte sur la taille des haies et des bosquets, le curage des fossés et l'épandage des boues résiduaires, la création et le balisage des chemins de randonnées pédestres ou équestres, l'entretien des berges des cours d'eau et des étangs, le retardement d'une récolte dans l'attente de l'arrivée à maturité d'une couvée ou de la germination d'une plante rare, l'installation d'ouvrages destinés à recevoir des nids d'échassiers ou de rapaces, l'élevage dans un cadre naturel auprès de sa clientèle, la mise en place de cultures à gibier, l'occupation
} 
Dans cette perspective, la politique d'aménagement du territoire doit être pensée à partir du prix de la terre qui doit redevenir le critère d'affectation du capital foncier et l'instrument privilégié des politiques en faveur de l'environnement rural et des équilibres économiques inter-régionals. Pour cela, il faut dissocier la foncier de l'agricole sans pour autant passer d'un extrême à l'autre et ignorer l'importance de l'activité agricole dans l'aménagement de l'espace rural.

\section{CONCLUSION}

Nous pouvons donc affirmer que tout converge pour corroborer la thèse selon laquelle la politique agricole commune s'explique aussi par les objectifs fonciers de la profession agricole.

Nous pouvons, aussi, supposer que cette politique a des effets pervers en matière d'aménagement du territoire rural parce qu'elle limite largement le redéploiement des usages du sol autour de ses valeurs de consommation.

Nous pouvons, enfin, soupçonner que le soutien de la valeur vénale de la terre est une meilleure hypothèse de travail pour comprendre les politiques d'aménagement du territoire rural que le traditionnel objectif d'équilibre inter-régional. La terre reste l'une des ressources les plus abondantes des espaces ruraux. Elle est pour cette raison un facteur incontournable de son développement. L'économie des espaces ruraux devient, dans cette perspective, une économie des règles qui gèrent le capital foncier et ses modes d'appropriation. L'importance des droits à construire et des droits à produire dans la valeur d'un terrain nous incitant à penser que les politiques de zonage, le droit rural, la fiscalité foncière, etc. sont au service de ceux qui les manipulent. Toutes ses réglementations publiques du marché de la terre servent à la fois à organiser le contrôle politique de l'espace communal, à produire des occasions de valorisation de son capital foncier et à limiter l'entrée de l'argent anonyme. Une étude détaillée des effets de cette politique nous permettrait de montrer que le contrôle politique du marché de la terre n'est peut être pas le meilleur moyen de valoriser son capital foncier. S'interroger sur l'importance du propriétaire dans la relation bailleur-preneur, sur les effets de la fiscalité foncière sur le comportement des acteurs et sur les effets de la politique de zonage sur le redéploiement des usages du sol sont des thèmes susceptibles d'accréditer nos soupçons.

\section{Bibliographie:}

[1] Alston J.M. (1986), «An analysis of growth of U.S. farmland prices 1963-1982», American Journal of Agricutural Economics, 68, 1, pp.1-9.

[2] Balisacan M. et Roussamet J.A. (1987), "Public choice of economic policy: the growth of agricultural protection », Weltwirtschaftliches Archiv, 123, 2, pp.232-248.

[3]Boinon et Cavaihles J. (1988), «Essai d'explication de la baisse du prix des terres, dans « La terre succession et héritage », Etudes Rurales, 110-111-112, Ed. EHESS, Paris, Avril-décembre.

[4]Brun A., Cavaihles J., Perrier-Cornet P. et Schmitt B. (1992), «Les espaces ruraux revisités », Revue d'Economie Régionale et Urbaine, 1, pp.37-67.

[5]Cavailhes J. et Degoud S. (1995), «L'évaluation du prix des terres en France. Une application aux effets de la réforme de la PAC », Cahiers d'Economie et de Sociologie Rurales, 36, pp.49-78.

[6]Caziot P. (1930), «Le capital foncier et les capitaux d'exploitation», Revue d'Economie Politique, XLIV, pp.8-19.

[7] Delord B., Hairy D., Lacombe P., de la Villosoye (1994), Les concours publics à l'agriculture française: structures et évolution, Economie rurale, mars-juin, n²20-221, pp.196-191.

[8] Desriers M. (1996), 21 mai 1992: naissance de la nouvelle PAC, Les cahiers de l'Agreste, marsjuin, $\mathrm{n}^{\circ} 1-2$, Bilan de trois années d'application de la réforme, pp.7-12.

du terrain en vue d'éviter le retour à la friche ou de la lutte contre les risques d'incendies, ... (Gilardeau [1992], p.221). 
«Politique agricole et prix de la terre », Politiques et Management Public, 1997, décembre3, vol.15, numéro 4.

[9]Facchini F. (1995), «La qualité de l'environnement, nouvel enjeu de la réforme de la politique agricole commune », Revue Politiques et Management Public, 13, 1, mars, pp. 29-54.

[10] Gilardeau J.M. (1992), «De l'agriculture à l'environnement: contrats, initiatives privées », Revue de Droit Rural, 203, mai, pp.215-289.

[11]Greffe X. (1990), La valeur économique du patrimoine. La demande et l'offre de monuments. Economica, coll. Anthropos, Paris.

[12] Harris D.J.(1977), «Inflation-indexed price supports and land values », American Journal of Agricutural Economics, 59, 3, pp.489-495.

[13] Jung J. (1971), L'aménagement de l'espace rural, une illusion économique, Calmann-Lévy, coll. Perspectives de l'économique, Economie contemporaine, Paris.

[14] Lamaison P. (1988), « De la transmission à l'abandon », dans « La terre succession et héritage », Etudes Rurales, 110-111-112, Ed. EHESS, Paris, Avril-décembre.

[15] Leathers H.D. (1992), " « The market land and the impact of farm programs on farm numbers », American Journal of Agricutural Economics, 74, 2, pp.291-298.

[16] Le Cacheux J. et Mendras H. (1992), «Eléments pour une nouvelle politique agricole », Observations et diagnostics économiques. Revue de l'OFCE, 42, octobre, pp.95-134.

[17] Lemonnier E. (1994), Dix ans de quotas laitiers, Revue de droit rural, n²26, oct. pp.393-406.

[18] Levesque R., Kerisit R. et Oger X. (1994), La valeur foncière des quotas laitiers, Etudes foncières, $n^{\circ} 62$, pp.30-33.

[19] Marsh J. and al (1991), The changing role of the common agricultural policy, Belhaven Press, London and New-York.

[20] Melichar E. (1979), «Capital gains versus current income in the farming sector», American Journal of Agricutural Economics, 61, pp.1085-1092.

[21] Olson M. (1985) «Space, Agriculture and Organization », American Journal of Agricutural Economics, 67, 5, pp.928-937.

[22]Pope C.A. (1985), «Agricultural productive and consumptive use components of rural land values in Texas », American Journal of Agricutural Economics, 1, pp.81-86.

[23] Shalit H. et Schmitz A. (1982), «Farmland accumulation and prices », American Journal of Agricutural Economics, 64, 4, pp.710-719.

[24] Sneessens J.F. (1973), «Relation entre les prix des produits et le coût du facteur foncier en agriculture », Recherches Economiques de Louvain, Sept., pp.357-370.

[25] Thomson K.J. et Whitby M.C. (1976), «The economics of public acces in the countryside », Journal of Agricultural Economics, 27, 3, pp.307-320.

[26]Vantreese V.L., Reed M.R. et Skees J.R. (1986), « Mandatory production and asset values: a case of burley tabacco quotas », American Journal of Agricutural Economics, 71, 2, pp.319-325.

[27]Winyard H.(1984), « The corporate estate: implications of corporation in the agricultural sector », pp.111-121, Ed by M.L. Harrison, Corporatisme and the Welfare State, Gower University of Leeds. 